\title{
Hybrid Lasers Based on CdSe/CdS Core/Shell Colloidal Quantum Rods on Silica Microspheres
}

\author{
C. Grivas ${ }^{1, *}$, P. Andreakou ${ }^{1}$, P. Wang ${ }^{2}$, M. Ding ${ }^{2}$, G. Brambilla ${ }^{2}$, L. Manna ${ }^{3}$, and P. G. Lagoudakis ${ }^{1}$ \\ ${ }^{I}$ School of Physics and Astronomy, University of Southampton, Southampton SO17 1BJ, United Kingdom \\ ${ }^{2}$ Optoelectronics Research Centre, University of Southampton, Southampton SO17 1BJ, United Kingdom \\ ${ }^{3}$ Istituto Italiano di Tecnologia (IIT) I-16163 Genoa, Italy \\ chr.grivas@gmail.com
}

\begin{abstract}
Single-mode lasing at $\sim 628 \mathrm{~nm}$ above an absorbed pump power threshold of $67.5 \mu \mathrm{W}$, tunable within a $2.1-\mathrm{nm}$ range (30\% of the free-spectral-range) was obtained from colloidal $\mathrm{CdSe} / \mathrm{CdS}$ core/shell nanorods on whispering-gallery-mode silica microspheres.

OCIS codes: (140.3945) Microcavities; (160.4236) Nanomaterials
\end{abstract}

\section{Introduction}

Elongated colloidal core/shell semiconductor nanonorystals are attracting a great deal of interest as gain media due to a number of salient properties originating from their small size and the associated quantum confinement. These include low-threshold and temperature-insensitive lasing, reduced trapping of excited carriers, and the possibility to alleviate non-radiative Auger recombination effects by engineering the wavefunction distributions of the electrons, and holes within their volume [1]. Furthermore, their size and shape, and in turn electronic and optical properties, can be tuned using simple and cost-effective wet-chemistry methods, which also provide a wealth of possibilities for their incorporation into various types of micro- and nanocavities and material matrices.

Whispering-gallery-mode (WGM) microsphere resonators can have extremely high Q factors $\left(>10^{8}\right)$ and are therefore attractive for development of a variety of photonics devices such as miniature lasers, single-photon emitters, sensors and, as templates, for fundamental studies of light-matter interaction [2,3]. Here we report on tunable laser emission from silica microspheres coated with $\mathrm{CdSe} / \mathrm{CdS}$ core/shell nanorods whose lasing thresholds and modality are controllable by the pump coupling conditions.

\section{Fabrication and experimental details}

The nanocrystals were asymmetric, quasi-type-II heterostructures with a 3-nm-diameter spherical optically active CdSe core located at one end of the wider band-gap CdS rod. They were synthesized with the seeded-growth method [4], had an overall size of $27.734 \mathrm{~nm} \times 4 \mathrm{~nm}$ and exhibited a photoluminescence maximum at $610 \mathrm{~nm}$. Microspheres with diameters from 8 to $40 \mu \mathrm{m}$ and measured Q factors in excess of $10^{8}$ were produced by rotating an optical fiber taper (stem) and simultaneously heating its end with a $\mathrm{CO}_{2}$ laser beam. They remained attached to the stem and were coated by immersion in a toluene solution of the nanorods. The density of the nanorods attached on their surface was dependent on the nanorod concentration in the solution, the immersion time and the withdrawal speed. The spheres were pumped at wavelengths $\lambda \sim 400 \mathrm{~nm}$ with a tunable frequency doubled Ti:sapphire amplifier, operating at 250 $\mathrm{KHz}$ and emitting 180 -fs-short pulses with a linewidth of $5 \mathrm{~nm}$. The latter were evanescently coupled into the spheres using tapers with adiabatic transitions made of single-moded fibers at $405 \mathrm{~nm}$ and drawn with the heat-andpull technique. Laser signals were collected by either a fiber tip with a diameter of $\sim 50 \mathrm{~nm}$ or, the same taper used for pumping, and spectra were recorded with an optical spectrum analyser (OSA). The tip, made with a micropipette puller, was brought into contact with the sphere and moved on the surface along a meridian to the desired plane.

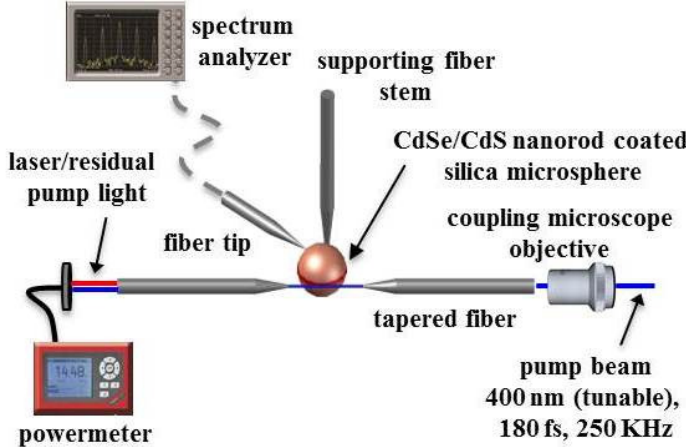

Fig. 1. Schematic of the experimental arrangement used for demonstration of fiber-coupled laser operation of $\mathrm{CdSe} / \mathrm{CdS}$ core/shell nanorods in silica

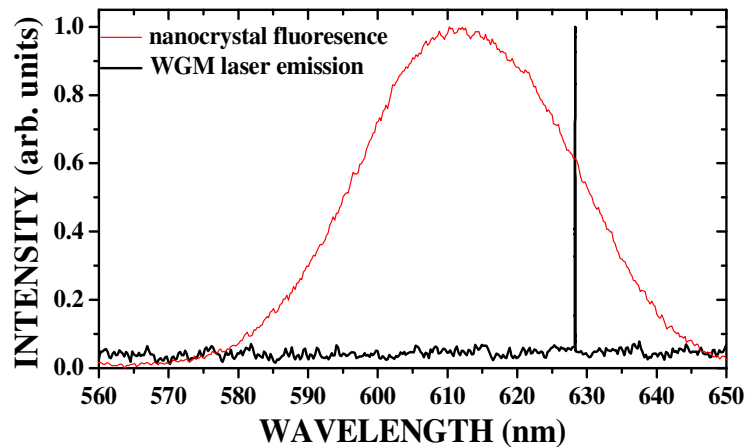

Fig. 2. Laser (black line) and fluorescence emission (red line) spectra from a $8-\mu \mathrm{m}$-diameter hybrid sphere and the $\mathrm{CdSe} / \mathrm{CdS}$ nanorods attached to the sphere, respectively 


\section{Laser operation}

A schematic of the experimental setup used is shown in Fig. 1. To optimize pump efficiencies, phase-matching of the propagation coefficients between the propagating mode in the taper and the fundamental WGM in the microsphere was established by suitably choosing the size of taper diameter with respect to that of the sphere [5]. For the microspheres studied, tapers with diameters ranging from 1 to $2 \mu \mathrm{m}$ were used for coupling. Fine tuning of the phase-matching conditions was achieved by adjusting the position of the latter along the taper waist. The microspheres were pumped slightly in the overcoupled regime in close proximity with the taper.

Figure 2 shows the laser spectrum for the single-WGM operation at $628.32 \mathrm{~nm}$ of a microsphere with a diameter of $8 \mu \mathrm{m}$. It was obtained by resonant pumping a fundamental WG pump mode with $|\mathrm{m}|=l$, where $m$ and $l$ are the angular momentum and the azimuthal numbers, above an absorbed pump power of $100 \mu \mathrm{W}$. Such modes are confined to the equatorial ring of the sphere (defined as the area parallel to the taper fiber axis perpendicular to the stem) and were identified by a dip observed in the transmission through the taper when the pumping wavelength was tuned. The laser line corresponds to the $1 \mathrm{~S}_{\mathrm{h}}-1 \mathrm{~S}_{\mathrm{e}}(\mathrm{CdSe})$ transition and its full-width-half-maximum (FWHM), as measured using an OSA at a spectral resolution bandwidth setting of 0.05 , was $\Delta \lambda=0.06 \mathrm{~nm}$. The linewidth obtained suggests a value of $\mathrm{Q}=(\lambda / \Delta \lambda) \sim 10^{5}$ for the coated microsphere. Microspheres with larger diameters produced multimode laser emission due to of the smaller free spectral range (FSR) $\Delta \lambda_{F S R}^{a z i n}$ in-between modes with different azimuthal numbers. Figure 3 shows the laser output power for the single-WGM operation of this microsphere, indicating a maximum output power of $5.5 \mu \mathrm{W}$ for $155 \mu \mathrm{W}$ of absorbed power, which corresponds to a slope efficiency of $6.4 \%$. Coupling of the pump beam to the resonator away from its equatorial zone, results in the appearance of a second set of laser-emission lines at lower wavelengths (Fig. 4), which corresponds to the $1 \mathrm{P}_{\mathrm{h}}-1 \mathrm{P}_{\mathrm{e}}$ (CdSe) transition. The 6.74-nm-spacing in-between these lines, corresponds to the $\Delta \lambda_{F S R}^{a z i n}$ of the hybrid resonator and is in agreement with a coated microsphere with a diameter of $8 \mu \mathrm{m}$.

The lasing wavelength, $\lambda_{\mathrm{L}}$ was tuned by heating with $3.5-\mu \mathrm{m}$-pulses of $200 \mathrm{fs}$ duration and $80 \mathrm{MHz}$ repetition rate from a tunable femtosecond laser, which were directed to the microsphere by a $4 \times$ microscope objective. The laser power was raised in successive steps and for each step the laser spectrum was recorded, indicating a $2.1 \mathrm{~nm}$ red-shift of the emission within the available power range (Fig. 5), which corresponds to $30 \%$ of the FSR at $\lambda_{\mathrm{L}}$. Since at $\lambda \sim 3.5 \mu \mathrm{m}$ the silica exhibits almost $100 \%$ absorption and the nanorods are transparent, the shift observed is attributed to changes of the sphere size and the refractive index nanorods (by heat conduction from the sphere).

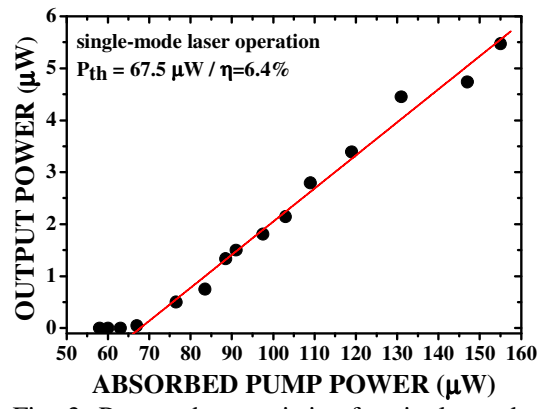

Fig. 3. Power characteristics for single-mode laser operation of a 8 - $\mu$ m-large microsphere.

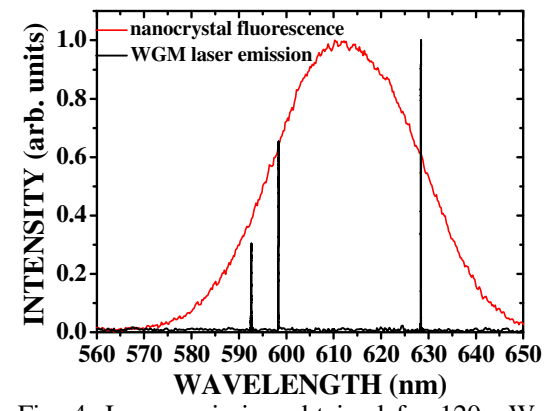

Fig. 4. Laser emission obtained for $120 \mu \mathrm{W}$ absorbed power by non-equatorial pumping.

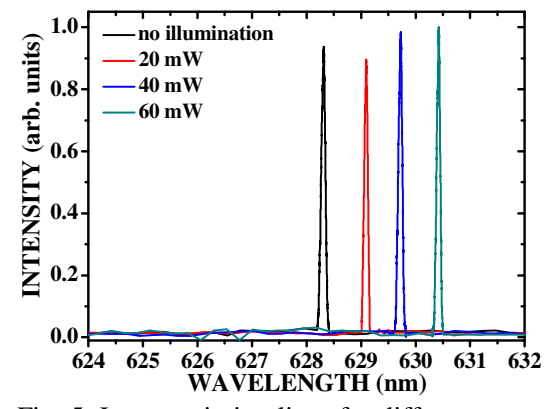

Fig. 5. Laser emission lines for different output powers of the $3.5 \mu \mathrm{m}$ laser used for heating

\section{Conclusions}

Operation of fiber-coupled hybrid lasers based on $\mathrm{CdSe} / \mathrm{CdS}$ quantum rods in silica microspheres was reported. The highly confined WGMs in resonators allowed for single mode emission above absorbed pump power threshold of $67.5 \mu \mathrm{W}$. The modality of the laser was tuned by varying the coupling conditions, pumping levels and the size of the microsphere template. Wavelength tunability of $2.1 \mathrm{~nm}$ was achieved by laser heating at a wavelength of $3.5 \mu \mathrm{m}$.

\section{References}

[1] R. Krahne, G. Morello, A. Figuerola, C. George, S. Deka, and L. Manna "Physical properties of elongated inorganic nanoparticles" Phys. Rep. 3-5, 75-221 (2011).

[2] J. Ward, O. Benson, "WGM microresonators: sensing, lasing and fundamental optics with microspheres" Laser Photon. Rev. 4, 553-570 (2011).

[3] Y.P. Rakovich, and J.F. Donegan. "Photonic atoms and molecules" Laser Photon. Rev. 4, 179-191 (2010).

[4] L. Carbone et. al. "Synthesis and Micrometer-Scale Assembly of Colloidal CdSe/CdS Nanorods Prepared by a Seeded Growth Approach" Nano Lett. 7, 2942-2950 (2007).

[5] J. C. Knight, G. Cheung, F. Jacques, and T. A. Birks, "Phase-matched excitation of whispering-gallery-mode resonances by a fiber taper" Opt. Lett. 22, 1129-1131 (1997). 